\title{
Congenital calcified unicuspid aortic valve with isolated aortic insufficiency without stenosis, and calcific retinal emboli
}

\author{
Alina Hasan ${ }^{1}$, Asif Mustafa $^{2}$, and Arnar Geirsson ${ }^{3}$ \\ ${ }^{1}$ Aga Khan University Hospital \\ ${ }^{2}$ Rush University Medical Center \\ ${ }^{3}$ Yale University School of Medicine
}

June 2, 2020

\begin{abstract}
Unicuspid aortic valve (UAV) is a very rare congenital anomaly with an estimated prevalence of 1 in 5000 in the adult population. It is often diagnosed by a 2D or 3D Transthoracic Echocardiogram (TTE) or Transesophageal Echocardiogram (TEE) [1]. Its usual presentation is in the third decade of life often as symptomatic aortic stenosis (AS). Rheumatic heart disease typically does not involve the aortic valve anatomically without also involving the mitral valve. Although the mitral valve may be diffusely abnormal anatomically, its function can be normal. Consequently, a patient with rheumatic heart disease can present initially with only aortic valve dysfunction, and therefore rheumatic heart disease has to be considered a cause of functionally isolated $\mathrm{AS} \pm$ aortic insufficiency (AI), or pure AI. We present a case of a 25-year-old male with a history of longstanding rheumatic fever and a recent embolic event to the left eye, who was seen in consultation for moderate AI and mobile calcification of the aortic valve with possible bicuspid valve by TEE. The echocardiogram also showed evidence of rheumatically deformed mitral valve with evidence of trace mitral regurgitation (MR). The patient was thought to have rheumatic valvular disease and planned for an aortic valve repair or replacement with a mechanical valve. During the surgery however, an UAV was found. It was replaced with a mechanical valve. The patient did well post-operatively and was discharged home five days later.
\end{abstract}

\section{CASE REPORT}

A 25-year-old male with chief complaint of dyspnea on exertion was seen in consultation for moderate AI and mobile calcification of the aortic valve with a complex past medial history including rheumatic fever diagnosed in 2016 and a recent embolic event to the left eye. Past surgical history was unremarkable. Relevant medications included Aspirin 325mg, Atorvastatin 20mg, Ibuprofen 600mg and Metoprolol 25mg. There was no family history of cardiac disease.

A TEE displayed a normal left ventricle function with an ejection fraction of 65-70\%, moderate AI and a bicuspid aortic valve. No AS was reported. However, a $0.9 \times 0.9 \mathrm{~cm}$ calcific non-mobile mass was noted to be adherent to the non/right coronary commissure (Fig 1). The mitral valve appeared rheumatically deformed with both mitral leaflets having restricted mobility. There was trace MR with a mean valve gradient of $2 \mathrm{~mm}$ $\mathrm{Hg}$ and calculated area of $2.2 \mathrm{~cm}^{2}$.

Because of moderate AI and mobile calcific mass on the aortic valve, along with a recent history of prior embolic event to left eye, the cardiac surgery was consulted for possible aortic valve repair or replacement.

The patient was taken to the operating room and midline sternotomy was performed, pericardium was opened and aortic, venous and cardioplegia cannulas were inserted. After the aorta was cross clamped and cardioplegia was given, the aorta was opened transversely. On examination, the aortic valve was unicuspid, Sievert type 2 with non-fusion of only the left and right cusps, and significantly calcified (Fig 2 ). The leaflets 
were excised and the annulus was decalcified. The root was irrigated with saline to ensure all calcium was evacuated. A 23mm On-X mechanical valve was selected. 2-0 Ethibond non pledgeted sutures were placed in non-everting fashion in order to place the valve in supra annular position. The valve seated nicely and sutures tied into place without any problem. After coming off bypass there was good function of the heart with no evidence of paravalvular leak with a mean gradient of $5 \mathrm{~mm} \mathrm{Hg}$ across the valve. The patient was in sinus rhythm and was closed up and brought to the ICU in stable condition.

Post-operatively, the patient did well. He was discharged on the fifth post-operative day on $2.5 \mathrm{mg}$ of warfarin in addition to his home medications.

\section{DISCUSSION}

The unicuspid aortic valve can be categorized into two types namely acommissural and unicommissural [4]. The former is pin-hole shaped with a small central orifice and is therefore associated with severe stenosis at birth needing early surgical repair. The latter has a slit-shaped appearance which has one attachment with an eccentric orifice that is usually larger allowing patients to remain asymptomatic until adulthood [4]. The latter was our case (Fig 2).

Malformation of the aortic valve leaflets causes an increased rate of calcification and fibrosis of the valve, progressing toward significant stenosis and/or regurgitation [5].

In a systematic review done on unicuspid aortic valve (UAV) in adults, it was found out that the most common lesion was isolated AS $(41 \% ; n=95)$ and AS with or without AI $(28 \% ; n=64)$ [6]. Our patient had no evidence of AS by echocardiogram, but rather had an isolated AI.

Dyspnea on exertion, angina, and few cases of monocular blindness due to retinal artery emboli, known as calcific retinal embolism (CRE), have been described as some presenting symptoms [7], as was with our case. CRE may also be the presenting feature of otherwise asymptomatic, clinically important underlying cardiovascular disease and, in particular, hemodynamically severe calcific valve stenosis [8].

UAV may not always be detected on echocardiography, but rather discovered on inspection after aortotomy as was in our case. UAV has an estimated incidence of $0.025 \%$ found by echocardiography and $5.5 \%$ found during aortic valve replacement [9]. Since there was significant history of longstanding rheumatic fever in our patient and the mitral valve seemed rheumatically deformed on echocardiogram with trace MR, the inability of echocardiography to pick up the unicuspid aortic valve made us associate the symptoms with the pathology of rheumatic valve disease. This was especially since rheumatic heart disease has to always be considered a cause of functionally isolated AS ( \pm AI) or pure AI with anatomically deformed mitral valve [3].

Symptomatic unicuspid valve warrants intervention. In adults, the most common treatment is aortic valve replacement [10]. Other interventions for repair for example, bicuspidization of the unicuspid valve, do exist. In fact, regurgitant UAV can be repaired successfully and reproducibly by converting it into bicuspid anatomy [11]; however, due to heavy calcification, valve replacement was the intervention chosen in our case.

\section{CONCLUSIONS}

UAV is a rare congenital finding and has two existing types with varying ages of presentation. An isolated AI without AS, as in our case, is an uncommon finding in UAV. History of rheumatic fever and evidence of mitral involvement on echocardiography can most likely sway the differential diagnosis to favor rheumatic valve disease in absence of unicuspid valve detection on echocardiography. Intervention once diagnosis of symptomatic unicuspid valve is made should be prompt and most commonly involves aortic valve replacement.

\section{FIGURE LEGENDS}

Figure 1 . Transesophageal echocardiogram showing what appears to be bicuspid aortic valve with heavy calcification between the non and right coronary cusps. 
Figure 2. Intraoperative picture showing heavily calcified unicuspid aortic valve with non-fusion of only the left and right cusps.

\section{REFERENCES}

1. Novaro, G., M. Mishra, and B. P Griffin, Incidence and echocardiographic features of congenital uniscupid aortic valve in an adult population. Vol. 12. 2003. 674-8.

2. Singh, S., et al., Unicuspid unicommissural aortic valve: an extremely rare congenital anomaly. Texas Heart Institute journal, 2015.42 (3): p. 273-276.

3. Roberts, W.C., Anatomically isolated aortic valvular disease. The case against its being of rheumatic etiology. Am J Med, 1970.49 (2): p. 151-9.

4. Falcone M, W., et al., Congenital Aortic Stenosis Resulting From a Unicommissural Valve. Circulation, 1971. 44 (2): p. 272-280.

5. Peacock, T., On Malformations of the human heart. John Churchill, London, 1858: p. 28-46, 75-76, 130-131, 139.

6. Mookadam, F., et al., Unicuspid Aortic Valve in Adults: A Systematic Review . Vol. 19. 2010. 79-85.

7. Sood, N. and C. Taub, Unicuspid aortic valve: an interesting presentation. Eur Heart J, 2008. 29 (10): p. 1295 .

8. Ramakrishna, G., et al., Calcific retinal embolism as an indicator of severe unrecognised cardiovascular disease. Heart (British Cardiac Society), 2005. 91 (9): p. 1154-1157.

9. Sitwala, P., et al., Unicuspid Aortic Valve - a Classic Presentation. Journal of Hospital Medicine, 2015. 10 (suppl 2) .

10. Nabati, M., et al., Unicuspid aortic valve in a middle-aged man: a rare case report. J Echocardiogr, 2014. $12(2)$ : p. $65-7$.

11. Schäfers, H.-J., et al., Bicuspidization of the Unicuspid Aortic Valve: A New Reconstructive Approach. The Annals of Thoracic Surgery, 2008. 85 (6): p. 2012-2018. 

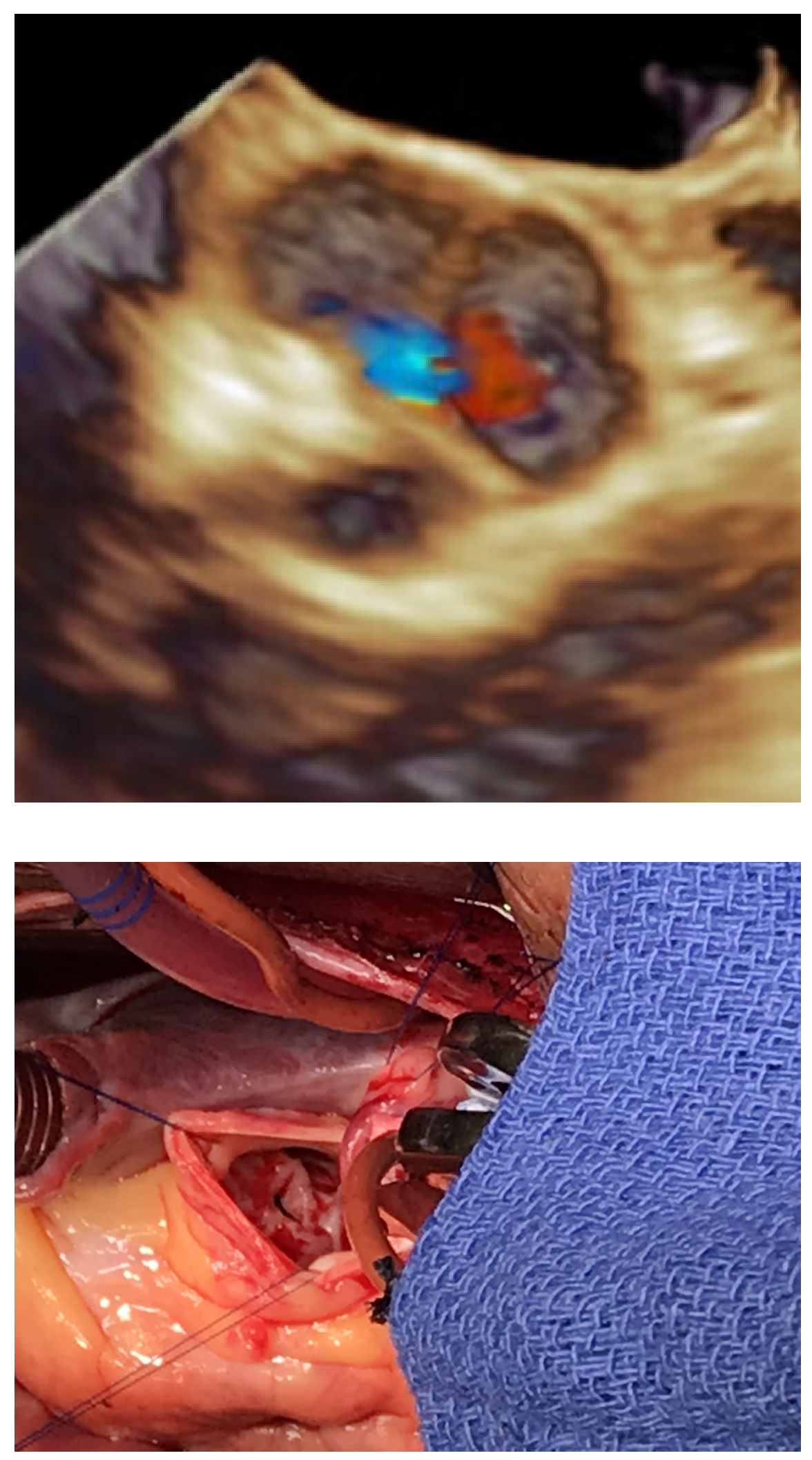